\title{
Associated and Polymerically Stabilized Dispersions
}

\author{
William B. Russel and Stacey Elliott \\ Department of Chemical Engineering \\ Princeton University \\ Princeton NJ 08544 USA \\ Robert A. Lionberger \\ Department of Chemical Engineering \\ University of Michigan \\ Ann Arbor MI 48109-2136 USA
}

\begin{abstract}
Our objective is to elucidate the relationships among interparticle forces, microstructure, and rheological properties for concentrated colloidal dispersions and associative polymer solutions. Here we address stable dispersions of spheres bearing grafted polymer chains and solutions of polymer with water soluble backbones modified by the addition of terminal hydrophobes. Non-equilibrium statistical mechanics, combined with treatments of micelles and brushes from the polymer physics literature, offers a means for confirming in detail the mechanisms suggested by recent experiments.
\end{abstract}

\section{REVIEW OF NON-EQUILIBRIUM THEORY}

Our description of a monodispersion of spheres addresses events on the diffusion time scale through a Smoluchowski equation that governs the distribution function for the configuration of all $N$ particles in the system ${ }^{1,2,3}$. Integration of the conservation equation for the $N$-particle distribution function $P_{N}$ over the positions of $N-2$ particles yields

$$
\frac{\partial P_{2}}{\partial t}+\nabla \cdot P_{2}\left\langle U_{1}-U_{2}\right\rangle_{2}=0
$$

CP469, Slow Dynamics in Complex Systems: Eighth Tohwa University International Symposium, edited by Michio Tokuyama and Irwin Oppenheim

(C) 1999 The American Institute of Physics 1-56396-811-8/99/\$15.00 
with

$$
\begin{gathered}
P_{2}\left\langle U_{1}-U_{2}\right\rangle_{2}=P_{2}\left\{E \cdot r+\langle C\rangle_{2}: E+\left\langle\frac{D}{k T}\right\rangle_{2} \cdot\left\langle F_{1}\right\rangle_{2}\right\}, \\
D=D_{11}+D_{22}-D_{12}-D_{21} \quad P_{2}\left\langle F_{1}\right\rangle_{2}=-P_{2} \nabla_{1} \Phi-\int \nabla_{3} \Phi P_{3} d x_{3},
\end{gathered}
$$

$r=x_{1}-x_{2}, \nabla=\nabla_{1}-\nabla_{2}$, and the no-flux boundary condition at contact. The relative velocity $U_{1}-U_{2}$ arises from interparticle, Brownian, and hydrodynamic forces that depend on the positions of all particles through the hydrodynamic tensors $C$ and $D_{i j}$, the total interparticle potential $\Phi_{i j}$, and $P_{N}$. We have decoupled conditional averages by expanding the diffusion tensor as the conditionally averaged pair diffusivity $\langle D\rangle_{2}$ plus deviations due to direct hydrodynamic interactions with a third particle, which are neglected. Explicit three-body couplings are retained only in the conditionally averaged total interparticle force $\left\langle F_{1}\right\rangle_{2}$. Therefore, the equations incorporate many-body hydrodynamic interactions in a mean field sense and require an explicit closure for the three-body thermodynamic couplings.

For Brownian particles suspended in a viscous fluid straightforward extension of pair interaction theories ${ }^{4}$, plus the decoupling approximation employed above for the hydrodynamic functions, leads to

$$
\Sigma=2 \eta^{H} E+\frac{1}{2} \int\left\{\left(1-\langle A\rangle_{2}\right) r \nabla \Phi-k T\langle W\rangle_{2} r r / r^{2}\right\} P_{2} d r
$$

The first term represents the hydrodynamic contribution, while the integral captures the effect of the pair potential and Brownian forces. The two scalar hydrodynamic functions, $A$ and $W$, characterize the component of $C$ along line of centers and its divergence, respectively.

Weak flows, having a small ratio of convection to diffusion such that $P e=$ $a^{2}|\boldsymbol{E}| D_{\mathrm{o}} \ll 1$ with $D_{\mathrm{o}}$ the Stokes-Einstein diffusion coefficient, only slightly perturb the equilibrium structure as

$$
P_{2}=n^{2} g(r ; \phi)\left\{1-\frac{a^{2}}{2 D_{o}} \frac{\boldsymbol{r} \cdot \boldsymbol{E} \cdot \boldsymbol{r}}{r^{2}} e^{i \omega t} f(r ; \phi)\right\},
$$

such that

$$
\left.\Sigma=2 \mu \boldsymbol{E} e^{i \omega t}\left\{\frac{\eta_{\infty}^{\prime}}{\mu}-\frac{9}{40} \phi^{2} \int_{[}^{[}\left(1-\langle A\rangle_{2}\right) s \frac{d}{d s} \frac{\Phi}{k T}-\langle W\rangle_{2}\right] f g s^{2} d s\right\} \equiv 2\left\{\eta^{\prime}-i \frac{G^{\prime}}{\omega}\right\} E e^{i \omega t}
$$

In the zero frequency or low shear limit, the perturbation is in phase with the rate of strain and $f$ is real. The interparticle and Brownian forces then generate viscous stresses that add to the hydrodynamic component $\eta_{\infty}^{\prime}$ and determine the low shear viscosity $\eta_{0}$. In the high frequency limit $f$ is imaginary; so the interparticle and Brownian forces produce an elastic stress characterized by the shear modulus $G_{\infty}^{\prime}$ and the hydrodynamic contribution solely determines the high frequency limiting dynamic viscosity $\eta_{\infty}^{\prime}$. 
We approximate the effect of a third particle on the total interparticle force through a closure derived by Scherwinski ${ }^{5}$, who defined a "direct correlation force" $C_{2}$ by

$$
n^{2} C_{2}\left(r_{12}\right)=-P_{2} \nabla_{1} \Phi_{12}+n^{3} h\left(r_{12}\right) \int C_{2}\left(r_{13}\right) h\left(r_{23}\right) d x_{3}
$$

with $h=p-1$ and $p=P_{2} / n^{2}$ and coupled it to the total interparticle force through

$$
P_{2}\left\langle F_{1}\right\rangle_{2}+n P_{2} \int \nabla_{3} \Phi_{13} p\left(r_{13}\right) d x_{3}=n^{2} C_{2}\left(r_{12}\right)+n^{3} \int C_{2}\left(r_{13}\right) h\left(r_{23}\right) d x_{3},
$$

to obtain an approximation that reduces to the familiar hypernetted chain closure at equilibrium.

In the far field, the conditionally averaged pair diffusivity reduces to $\langle D\rangle_{2}=$ $2 D_{\mathrm{s}}{ }^{\circ}$, where $D_{\mathrm{s}}{ }^{\circ}$ is the short-time self-diffusion coefficient, since the spheres move independently in a mean field of other particles. The near field limit follows from the lubrication force between two particles in a quiescent fluid. We simply interpolate between these limits, either discontinuously for hard particles with weak interactions,

$$
\langle D\rangle_{2}=2 D_{s}^{o}\left[H(r-2 a) \frac{r r}{r^{2}}+\left(\delta-\frac{r r}{r^{2}}\right)\right]
$$

or with a piecewise continuous representation for other systems ${ }^{3}$. For the relative velocity in a shear flow, characterized by $\langle C\rangle_{2}$, a similar treatment works. The shorttime self-diffusion coefficient $D_{s}{ }^{\circ}$ and high frequency dynamic viscosity $\eta_{\infty}^{\prime}$ appearing here and in the stress are measurable quantities that depend only on the equilibrium structure. Quality data, as well as results from simulations, exist for disordered dispersions of hard spheres.

To test these closure approximations we calculated the linear viscoelastic responses for the hard sphere, Yukawa, and inverse power $\left(r^{-n}\right)$ potentials without hydrodynamic interactions ${ }^{2}$ and hard spheres with hydrodynamic interactions ${ }^{2}$. Comparison with results from computer simulations imposing no hydrodynamic interactions indicates the closure (6-7) to be qualitatively correct but reveals quantitative discrepancies akin to those observed for the hypernetted chain closure at equilibrium. Apparently the closure exaggerates the forces opposing deformation and, hence, predicts too small a perturbation from equilibrium. Nonetheless the predictions are quite robust to variations in the interparticle potential and volume fraction.

Comparison of calculations for hard spheres with hydrodynamic interactions against experimental data reveals the following ${ }^{3,6}$ :

(1) $G^{\prime}(\phi)$ in at least qualitative agreement with the data (Figure 1); 
(2) $\Delta \eta_{B}=\left(\eta_{o}-\eta_{\infty}^{\prime}\right) / \mu$ that conforms within $20-30 \%$ with the older body of data and captures qualitatively the divergence as $\phi->0.64$, but errs by $100 \%$ relative to more recent data (Figure 2);

(3) long-time self-diffusion coefficients, $D_{s}^{\infty} / D_{o}$, that conform to the data for $\phi<0.45$ and capture qualitatively the zero as $\phi->0.64$;

(4) non-equilibrium static structure factors for weak steady shear that exhibit the proper dependence on wave number but too small a magnitude; and

(5) stress-optical coefficients in the low shear limit with the proper dependence on volume fraction and roughly the right magnitude.

The qualitative agreement, but quantitative shortcomings, for the optical measures of structure, the low shear viscosities, and long-time self diffusion coefficients reinforce the assessment of the thermodynamic closure. Earlier results for the high frequency modulus indicate the hydrodynamic models to be quite satisfactory. Thus the theory is imperfect but robust and should suffice for mechanistic studies. With the exception of the light scattering measurements, a similar claim can be made for the formulation by Brady ${ }^{7}$, which totally ignores the thermodynamic effect of the third particle and produces similar stresses but quite different non-equilibrium structures.

\section{SPHERES WITH GRAFTED POLYMER LAYERS}

For spheres bearing grafted polymer layers we estimate the pair potential through the Alexander-de Gennes approximation, which views the polymer layers as chains of $N$ segments of length $\ell$ and excluded volume $v$ grafted at density $\sigma$. Minimizing a mean field form for the free energy yields the layer thickness as $L=(v \sigma / 6 \ell)^{1 / 3} N \ell^{3}$ for highly stretched chains in good solvents, as opposed to $N^{1 / 2} \ell$ for Gaussian coils. The increased free energy upon compressing two layers to separations less than $2 L$ comprises the interaction potential, which the Derjaguin approximation converts to spheres bearing thin polymer layers. Monte Carlo simulations then determine the equilibrium pair distribution function.

To obtain the requisite hydrodynamic functions, flow in the polymer layer modeled through the Brinkmann equation is coupled with Stokes flow in the gap to obtain the lubrication limit ${ }^{8}$. The far-field requires the high frequency viscosity or short-time self-diffusion coefficient, so we combine mean field and asymptotic approximations that offer reasonable, though still untested, estimates. The most important consequence is reflected by the relative velocity along lines of centers in a shear flow, plotted for a range of volume fractions in Figure 3 . Note that the behavior changes from that of hard spheres with radius $a+L$ for $\phi_{\mathrm{eff}}=0.40$, due to a non-draining polymer layer, to hard spheres of radius $a$ for $\phi_{\mathrm{eff}}=0.74$, indicating an effectively freedraining polymer layer.

Calculations of the high frequency modulus as a function of particle volume fraction $\phi$ and ratio of layer thickness to radius, $L / a$, reveal a couple of interesting features': 
(1) a substantial reduction in the modulus with decreasing permeability of the layer for $\phi(1+L / a)^{3}<0.64$ but a minimal effect above close packing of the polymer layers and

(2) a variation in modulus with softness of the layer, i.e. $L / a$, that strongly resembles data for PMMA spheres stabilized by poly(hydroxy stearic acid) ${ }^{10}$.

The first arises from the transition in the hydrodynamic functions noted above and establishes the validity of the common practice of inverting measurements of the modulus to obtain the interaction potential, but only for concentrations beyond close packing. At these conditions the very high viscosity of the medium pushes the pair together and overwhelms the lubrication force controlled by the relatively low solvent viscosity. Similar calculations, but with the force between poly(hydroxystearic acid) layers on mica ${ }^{9}$, suggest the density of adsorbed polymer chains on the particles to be about a factor of three lower than on the mica surfaces. Therefore, the interparticle potential was adjusted and calculations performed for both the high frequency modulus and the steady shear viscosities.

Figures 4 (a) and (b) compare the results of these calculations for the high frequency modulus with the data of Mewis and coworkers ${ }^{10}$ for several different values of $L / a$ and $L / \zeta_{0}$ ranging from free-draining to highly non-draining. Note the qualitative difference in the volume fraction dependence, i.e. the curvature on this semi-log plot, for volume fraction below and above random close packing, i.e. $\phi_{\mathrm{eff}}=0.64$. The modulus is sensitive to the permeability of the layer below this transition, but is relatively insensitive above. Also the data parallels the solid curve, which neglects hydrodynamic interactions for $\phi_{\text {eff }}>0.64$. Thus, the conclusion above still stands, though the softer potential permits spheres to approach to smaller separations and, therefore, requires a somewhat larger volume fraction to suppress the effect of hydrodynamic interactions. As the data still lies consistently below the predictions, one might conclude that the potential employed in the calculations is still a bit too strong.

The low shear viscosities in Figures 5 derive from setting $f(r, \phi)=f(r, 0)$, i.e. neglecting entirely the higher order terms in the equation governing the non-equilibrium structure. The data and the calculations are plotted against the core volume fraction and virtually all correspond to $\phi_{\mathrm{eff}}<0.64$. Consequently, the predictions are insensitive to the softness of the potential or the permeability of the layer, primarily sensing the range, associated with the thickness of the polymer layer. The permeability affects the viscosity, with the lower values producing a more faithful representation of the data.

From this we conclude that the high and low frequency limits of the linear viscoelastic response can be predicted at least semiquantitatively from the nonequilibrium theory with rather simple approximations for the interparticle potential, hydrodynamic interactions, and many-body couplings. Knowledge of the interaction potential and the permeability of the layer is critical, however, and generally incomplete. 


\section{MICELLAR SOLUTIONS}

Associative polymers, in the form of hydrophobically modified water soluble polymers, serve as rheology modifiers in paints, adhesives, mastics, detergents, shampoos, agricultural sprays, asphalts, and a variety of other materials. Each combines soluble and insoluble components in a telechelic, comb graft, star block, or alternating/random copolymer architecture and can exhibit gas-liquid, percolation, and gelation transitions. The solutions often exhibit remarkable viscoelasticity including extremely high (but Newtonian) low shear viscosities, shear and extensional thickening, precipitous shear thinning, and large elastic recoveries ${ }^{11}$.

Our model associative polymers consist of $35 \mathrm{~kg} /$ mole poly(oxyethylene) linked via monoisocyanates to $C_{16}$ and $C_{18}$ alkane endcaps and display somewhat surprising behavior. The first surprise was an entropically driven phase transition with coexistence between a dilute micellar "gas" and a highly associated micellar "liquid", as partially anticipated by Semenov and coworkers ${ }^{12}$ for flowerlike micelles with high aggregation numbers in a good solvent. Characterization of the dilute (gas) phase dynamic by light scattering and capillary viscometry yielded the micellar diameters $d=35-40 \mathrm{~nm}$ and aggregation numbers $p=20-35$ from the intrinsic viscosity and the strength of the attraction $1 / \tau=10-15$.

The associated liquid phase displays the linear viscoelasticity now routinely expected for these telechelic/triblock copolymers and normally interpreted through the reversible network theory ${ }^{13}$. However, closer comparison with our data reveals significant deviations from this model, which assumes a homogenous solution with all chains bridging between junctions (micelles) and predicts the high frequency modulus as $G_{\infty}^{\prime}=n k T$, i.e. increasing linearly with chain density $n$. Our measurements instead exhibit a roughly quadratic increase with effective volume fraction $\phi_{p}=0.4[\eta]_{p} n$ for $0<\phi_{p}<1$. For $\phi_{p}>1 G_{\infty}^{\prime} / n k T$ is roughly constant, but still varies with $p$.

This suggests viewing the micelles as colloidal particles interacting through an equilibrium pair potential $\Phi$, for which the non-equilibrium theory without hydrodynamic interactions predicts

$$
G_{\infty}^{\prime} \cong \frac{N}{d} \frac{d^{2} \Phi}{d r^{2}} \phi_{p}
$$

with $N$ the number of nearest neighbors and $r$ the center-to-center distance. The thermodynamics suggests $N=2 \phi_{p} / \tau$ and $d^{2} \Phi / d r^{2} \approx p^{3 / 2} k T / d^{2}$, indicating that the stress should scale as

$$
G_{\infty}^{\prime} \cong \frac{p^{3 / 2} k T}{\tau d^{3}} g\left(\phi_{p}\right)
$$

This scaling successfully collapses the data for the two polymers examined (Figure 6) and motivates more detailed modeling. 
To develop a quantitative model for the intermicellar potential arising from excluded volume interactions we extended the variational approach of $\mathrm{Ho}$ and Witten ${ }^{17}$ to compressed chains and added the attraction due to bridging chains, yielding micelle radii and a depth for the attractive minima that agree well with the data. The Brinkmann model for flow in the corona of the micelle provides hydrodynamic mobilities from the porous sphere solutions of Felderhof ${ }^{18}$. With this input the Monte Carlo simulations and the calculations of the linear viscoelasticity are straightforward up to $\phi_{p}=1$. The results in Figure 6 fall above the data for values of the permeability consistent with the blob size associated with the stretched chains. The fault may lie assumption of strong stretching in the theory leading to the interaction potential, whereas the modest aggregation numbers of the micelles may fall short of that limit. The fact that the predictions for $p=33$ are closer than those for $p=20$ supports this hypothesis. Beyond $\phi_{p}=1$ the physics changes, since the highly stretched chains form a semi-dilute solution and relax somewhat due to the additional screening. This effect has not yet been incorporated into the theory.

\section{SUMMARY}

Non-equilibrium theory with approximations for the interparticle potential from fundamental theory and hydrodynamic interactions from a combination and mean-field and lubrication theories offers physically reasonably predictions for the linear response of dispersions and solutions interacting via polymer brushes. At this point even the crudest of treatments of the many-body couplings through the pair potential yields representations that are quantitatively consistent with experiments.

Acknowledgement This research was supported by the Program in Multiphase Processes within the Division of Engineering of the National Science Foundation.

\section{References}

1. R.A. Lionberger \& W.B. Russel, J. Rheol. 38, 1885 (1994).

2. R.A. Lionberger \& W.B. Russel, Journal of Chemical Physics 106, 402-16 (1997).

3. R.A. Lionberger \& W.B. Russel, J. Rheol. 41 399-425 (1997).

4. W.B. Russel, D.A. Saville \& W.R. Schowalter, Colloidal Dispersions, Cambridge University Press, 1989.

5. K. Scherwinski, Mol. Phys. 70,797 (1990).

6. R.A. Lionberger \& W.B. Russel, Adv. Chem. Phys. (in press).

7. J.F. Brady, J. Chem. Phys. 99, 567 (1993).

8. A.A. Potanin \& W.B. Russel, Physical Review E 52, 730-37 (1995).

9. S.L. Elliott \& W.B. Russel, J. Rheol.42 (2) (1998).

10. W.J. Frith, T.A. Strivens, \& J. Mewis, J. Colloid Interface Sci. 139, 55 (1990); J. Mewis \& P. D'Haene, Makromol. Chem. Macromol. Symp. 68, 213 (1993).

11. M.A. Winnick \& A. Yekta, Current Opinion in Colloid and Interface Science 2 424-436 (1997). 
12. A.N. Semenov, J.-F. Joanny \& A.R. Khokhlov, Macromolecules 281066 (1995).

13. F. Tanaka \& S.F. Edwards, J. Non-Newt. Fluid Mech. 43 247-309 (1992).

14. H. Li \& T.A. Witten, Macromolecules 27, 449-457 (1994).

15. P. Reuland, B.U. Felderhof \& R.B. Jones, Physica A 93 465-475 (1978).

16. Q.T.T. Pham, "Associative Triblock Copolymers in Solution and Dispersions", PhD Dissertation, Department of Chemical Engineering, Princeton University, 1998.

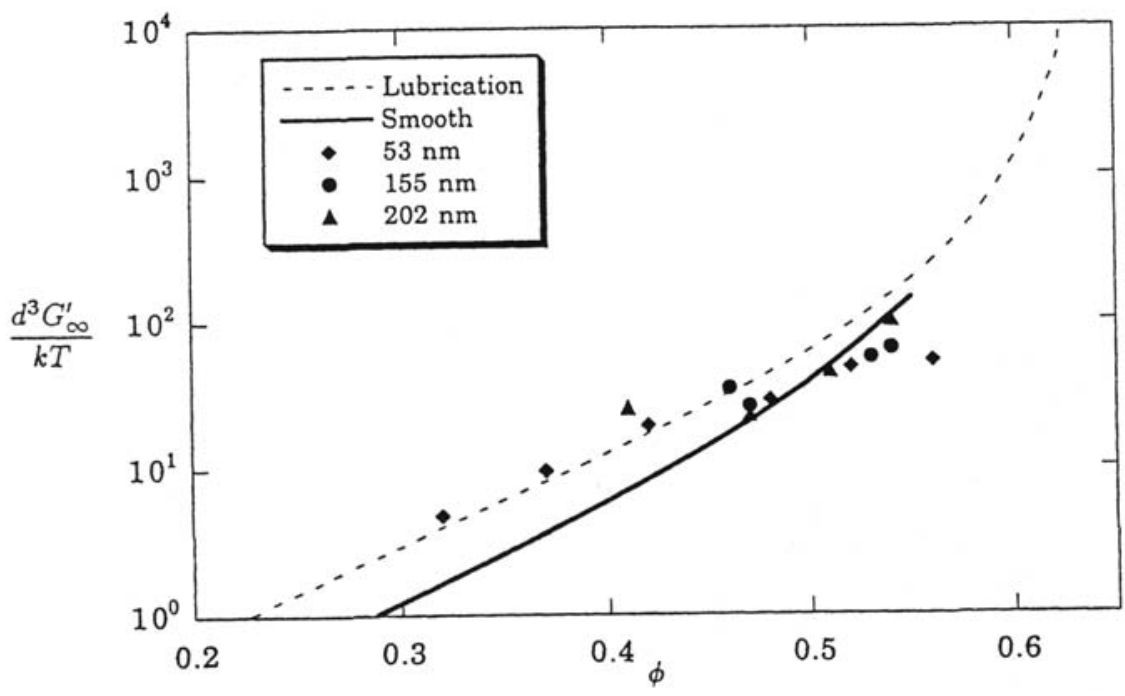

FIGURE 1: High frequency modulus of hard spheres as a function of volume fraction compared with data from hard sphere silica dispersions [6]. 


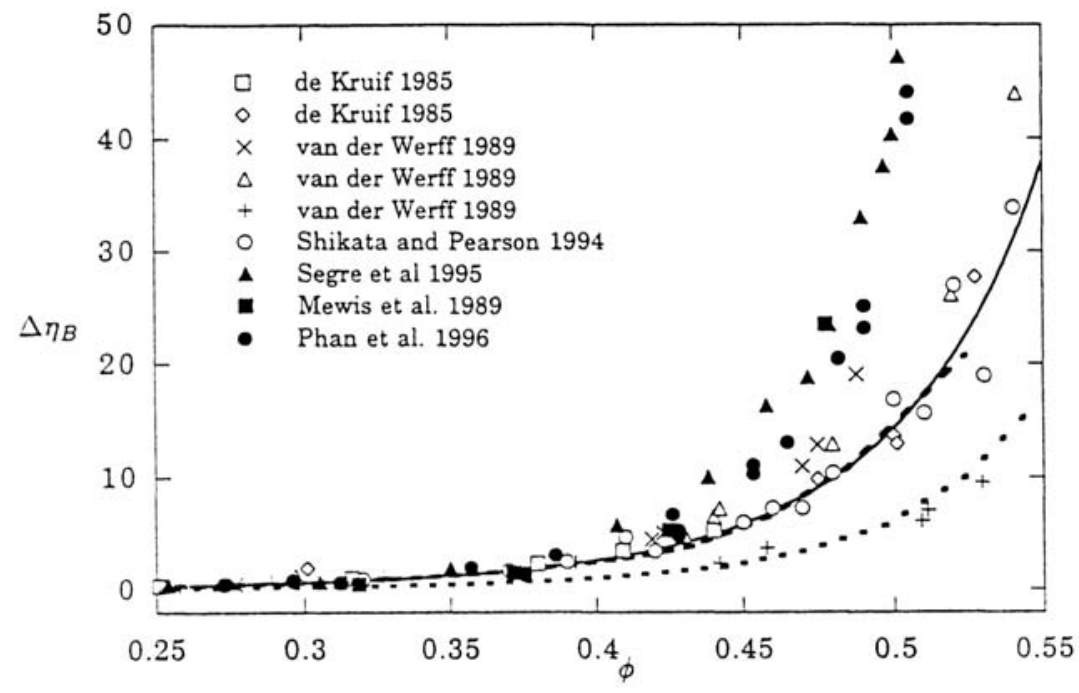

FIGURE 2: The Brownian contribution to the low shear viscosity compared with that extracted from all the experimental data available [6].

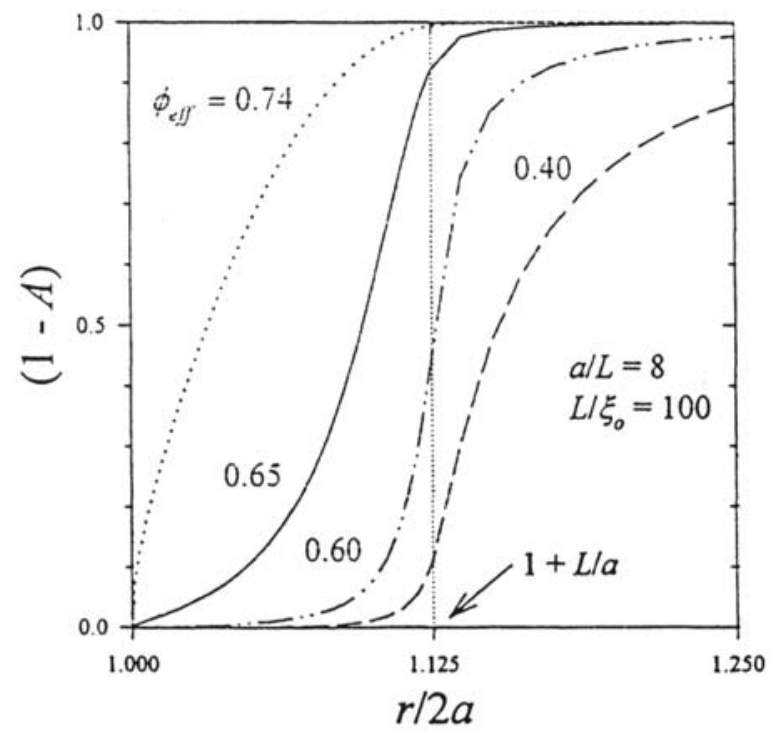

FIGURE 3: The magnitude of the relative velocity along lines of centers as a function of center-to-center distance for various volume fractions for $a / L=8$ and $L / \xi_{0}=100$ [6]. 

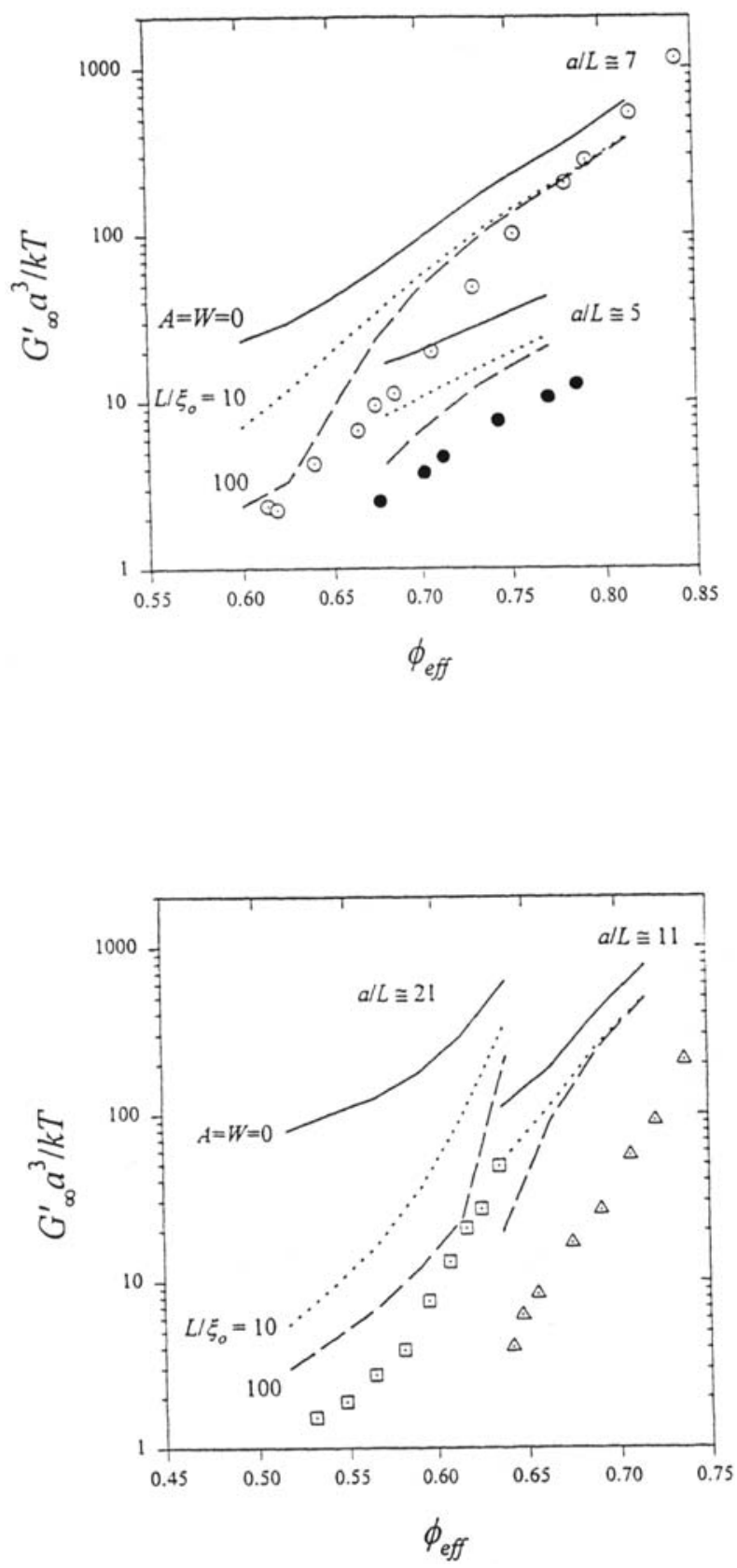

FIGURE 4: The dimensionless high frequency shear modulus as a function of the effective volume fraction for several different values of $L / \xi_{0}$ compared with data from Mewis and coworkers [10] for $a / L=5$ and 7 (a) and 11 and 21 (b) [6]. 


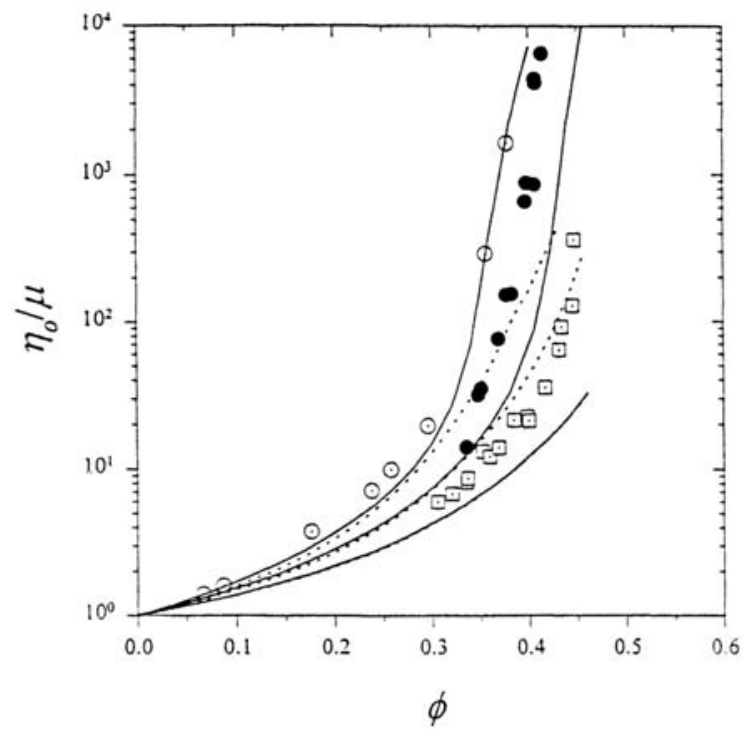

FIGURE 5: Low shear viscosity as a function of core volume fraction for $a / L=5,11$, and 21 (top to bottom) and $L / \xi_{0}=10(--)$ and $100($ compared with data of Mewis and coworkers [6].

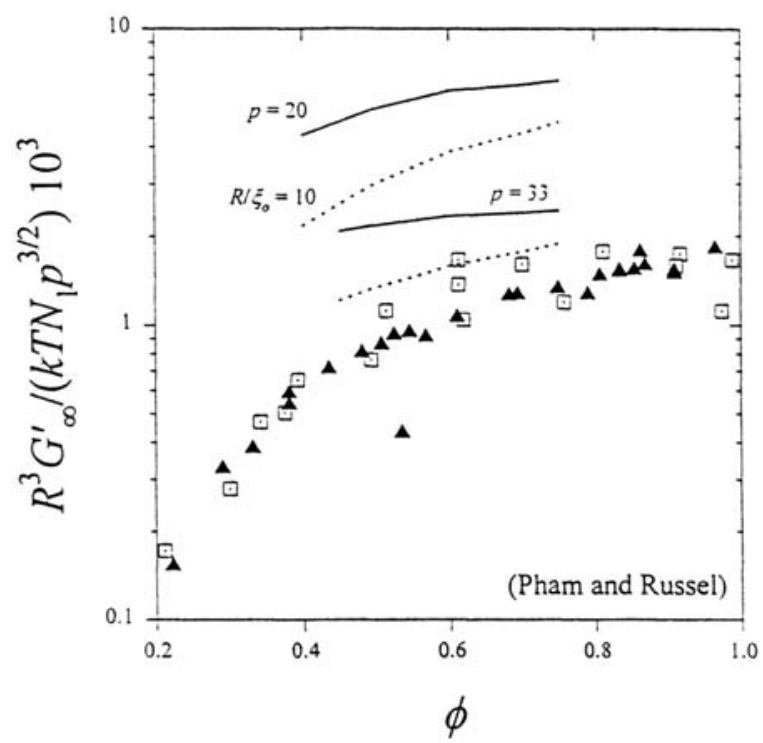

FIGURE 6: Normalized shear modulus for micellar solutions of triblock copolymers with $p=20$ and 33 as a function of effective volume fraction compared with data of Pham [16]. 